\title{
WEIGHTS BASED DECISION LEVEL DATA FUSION OF LANDSAT-8 AND SENTINEL-1 FOR SOIL MOISTURE CONTENT ESTIMATION
}

\author{
Oualid Yahia \\ Surrey Space Centre \\ University of Surrey, United Kingdom \\ o.yahia@surrey.ac.uk
}

\author{
Raffaella Guida \\ Surrey Space Centre \\ University of Surrey, United Kingdom \\ r.guida@surrey.ac.uk
}

\author{
Pasquale Iervolino \\ Surrey Space Centre \\ University of Surrey, United Kingdom \\ p.iervolino@surrey.ac.uk
}

\begin{abstract}
A novel decision level data fusion algorithm for soil moisture content estimation is proposed in this paper. Firstly, individual estimations are determined, respectively, from the inversion of the Integral Equation Model (IEM) for Sentinel-1 and from the Temperature Vegetation Dryness Index (TVDI) for LANDSAT-8. Then, a feature level fusion of these methods is performed using an Artificial Neural Network (ANN). Finally, all estimations including the feature level fusion estimation are fused at the decision level using a novel weights based estimation. The area of interest for this study is Blackwell Farms, Guildford, United Kingdom and datasets were taken on 17/11/2017 for both Landsat-8 and Sentinel-1. Estimation from the proposed decision level fusion method produces a Root Mean Square Error RMSE (1.090\%) which is lower than RMSE of the individual estimations of each sensor as well as that of the feature level fusion estimation.
\end{abstract}

Index Terms - data fusion, weights based, Integral equation model, Temperature vegetation dryness index, Artificial Neural Network.

\section{INTRODUCTION}

An accurate Soil moisture content (SMC) estimation can lead to a better understanding of land surface conditions, natural resources managements (especially in agriculture) and the different interactions in land-atmosphere system. The most accurate SMC estimation is achieved by the direct measurement of SMC using handheld or in-situ sensors. The latter are limited to discrete measurements (point-based) at specific locations which is expensive in terms of both time and effort and do not represent the spatial distribution and the variability of SMC [1]. That is the reason why remote sensing (indirect measurements) are starting to gain some footing as a more viable and less expensive option. Remote sensors like Synthetic Aperture Radars (SAR) are especially known for their huge potential for SMC estimation at the regional and global scales [2]. Numerous literature has determined that SMC estimations can also be achieved (with variable degrees of accuracy) using a synergy of space borne multispectral and thermal infrared sensors [3]. Each of the aforementioned sensors can bring something different to the table. Multispectral imagers are characterised by high spatial resolution, a wide range of satellites to choose from, and robustness to the effect of partial vegetation cover. Thermal infrared imagers share the same properties expect they have a lower spatial resolution than their multispectral counterparts. However, multispectral imagers do not offer any information in night time and both multispectral and thermal infrared imagers suffer in poor weather conditions (clouds), not to mention that both cannot offer any surface penetration. On the other hand, a SAR offers very good spatial resolution, allweather all-light datasets, and surface penetration. SAR is also extremely sensitive to surface roughness and intense vegetation covers. It is important to highlight that all these sensors suffer in terms of temporal resolution. Data fusion techniques have been a popular solution to mitigate the limitations of each sensor due to their ability to combine information gathered from different sensors to achieve better understanding (far better than that which is attainable by a single sensor) of an object or phenomenon. Data fusion can be performed at different levels of processing: Signal Level fusion, image level fusion, feature level fusion and decision level fusion [4], this study is interested in the last two. In this study, an SMC estimation using decision level data fusion is proposed with the objective of achieving better accuracy than that of a single sensor. The inner workings of this methodology are deeply explained in the methodology section.

\section{METHODOLOGY}

This study proposes a weight based decision level data fusion method for SMC estimation. In order to fully explain how the fusion scheme works, a presentation of the individual estimation methods is necessary. The estimation methods in question are the Integral Equation Model (IEM) inversion for SAR and the Temperature Vegetation Dryness Index (TVDI) for the synergy of the multispectral and thermal infrared. This section would offer a brief description of these methods and an explanation of how the two independent estimations are fused at feature level. More details on the application of both methods are contained in [5]. Finally, the novel decision level fusion framework is introduced. 


\subsection{Integral Equation Model}

The backscattered SAR signal is influenced by numerous surface characteristics such as surface roughness, dielectric features of the soil and soil moisture content levels, and radar characteristics such as the frequency, incidence angle and polarization. The dielectric features of the soil are referred to as the dielectric constant $\left(\varepsilon_{\mathrm{s}}\right)$. The latter is heavily influenced by the mineralogical composition of the soil as well as the SAR frequency and soil moisture content presence. A regression analysis determined that this dependence is of polynomial nature [6]. That analysis yielded individual polynomial expressions for $\varepsilon_{\mathrm{s}}$ as a function of the soil moisture content for each frequency and soil type. The Integral Equation Model (IEM) is a single scattering theoretical model represented by a complex mathematical expression which inversion allows the estimation of soil moisture content levels and surface roughness parameters. The accuracy of SMC estimation using IEM inversion suffers in medium to intense vegetation covers. Moreover, the IEM model tends to underestimate the radar response in $\mathrm{C}$-band in presence of vegetation, which would have a huge effect on the accuracy of SMC estimation. Translating this result in requirements, it means that the IEM inversion estimation is only reliable when the Normalized Difference Vegetation Index $(N D V I) \leq 0.2$ [7]. This is enough reason to pursue a different estimation using a different sensor to deal with this issue.

\subsection{Temperature Vegetation Dryness Index}

Land Surface Temperatures (LST) and NDVI of heterogonous areas can be represented by a two-dimensional scatter plot which could be a triangular feature space as in [8]. There, the changes in SMC levels (described within the LST/NDVI triangle) are plotted as a function of surface temperature and fractional vegetation cover (for which NDVI is a good metric). The differences in radiative temperatures between soil and various vegetation canopies have different effects on LST. Evapotranspiration is another factor having an influence on LST through the energy balance of the surface: the available energy for sensible heating of the surface increases whenever there is a decrease in evapotranspiration due to stomatal resistance to transpiration which is controlled by soil moisture availability. The authors in [8] proposed an index that describes the $L S T / N D V I$ feature space called Temperature Vegetation Dryness Index (TVDI). TVDI values range from 0 to 1 , indicating respectively low and high levels of SMC. In [8], authors established that SMC and TVDI have a linear relationship. A more comprehensive review of this method with the description of its physical properties is found in [8].

\subsection{Artificial Neural Networks (ANN)}

Given the aforementioned mathematical complexity of the IEM, calculating SMC by its inversion is a hard and complex task. That motivated authors in [9] to use an Artificial Neural Network known as a multi-layer perceptron (MLP) for the inversion process. MLP is a system inspired by human neurons; it consists of artificial neurons in the form of units connected to each other by weights. The structure of an ANN has 3 layers: input layer, hidden layer and an output layer. All MLPs implemented here consist of an input layer in the form of a vector composed of different features, one or more hidden layers, and an output vector containing the ground measured SMC values. In this study, 3 different ANNs are used. The first one is used for the IEM inversion and it is called ANN $\mathrm{ANM}_{\text {IEM }} \mathrm{A}$ second $\mathrm{ANN}$, $\mathrm{ANN}_{\text {TVDI}}$, uses TVDI as an input while the third one, $A N N_{F L F}$, is used for feature level fusion. Each of these ANNs has its own input feature vector. $\mathrm{ANN}_{\mathrm{IEM}}$ has an input vector composed of the RMS height $(s)$ and correlation length $(l)$ of the soil, backscattering coefficient $\sigma^{\circ}$ and the incident angle $\theta_{i}, \mathrm{ANN}_{\mathrm{TVDI}}$ has an input vector composed of TVDI values only and the input of $A \mathrm{AN}_{\mathrm{FLF}}$ is explained in the following subsection.

\subsection{Feature level fusion}

In the feature level fusion, salient features are extracted from each sensor in question and then concatenated to create what is called joint feature vector. Let $X=\left\{x_{1}, x_{2} \ldots x_{m}\right\}$ and $Y=$ $\left\{y_{1}, y_{2}, \ldots y_{n}\right\}$ denote feature vectors $\left(X \in R^{m}\right.$ and $\left.Y \in R^{n}\right)$ representing information obtained via the two different sources. Vectors $X$ and $Y$ are merged to generate a new feature vector $Z$ for better representation and potentially better estimation of the observed phenomenon [10]. In this study, the feature vectors to be merged into a joint feature vector are input vectors of $\mathrm{ANN}_{\mathrm{IEM}}$ and $\mathrm{ANN}_{\mathrm{TVDI}}$. Authors in [11] used somewhat of a similar approach where (NDVI, thermal infrared temperature, $\theta_{i}, \sigma^{\circ}$ ) was the input vector of a nonlinear ANN used to infer $S M C$ to support a hydrological simulation studies. However, in this study, the joint feature vector is composed of $\left(s, l, \theta_{i}, \sigma^{\circ}, T V D I\right)$ instead, the latter represents the input vector to $\mathrm{ANN}_{\mathrm{FLF}}$. Initially, the training phase links the previously mentioned input vector to the measured $S M C$ values. The training set $\left(s, l, \theta_{i}, \sigma^{\circ}, T V D I\right.$, $S M C$ ) is the input of the neural network where the first 5 parameters of each set are used to calculate the last one SMC. The used $\mathrm{ANN}_{\mathrm{FLF}}$ has one hidden layer (composed of 10 nodes) and the training method is Levenberg-Marqurdt. Out of the 110 data samples available, $80 \%$ were used for training, $10 \%$ for validation and $10 \%$ for testing. The size of the hidden layer and the training samples division were determined after numerous experimentations and this particular configuration seems to yield the best results in terms of accuracy. 


\subsection{Decision level fusion}

The novelty of this methodology lies in the addition of feature level fusion estimation instead of just using the sensors in question. The decision level fusion scheme is depicted in the figure 1:

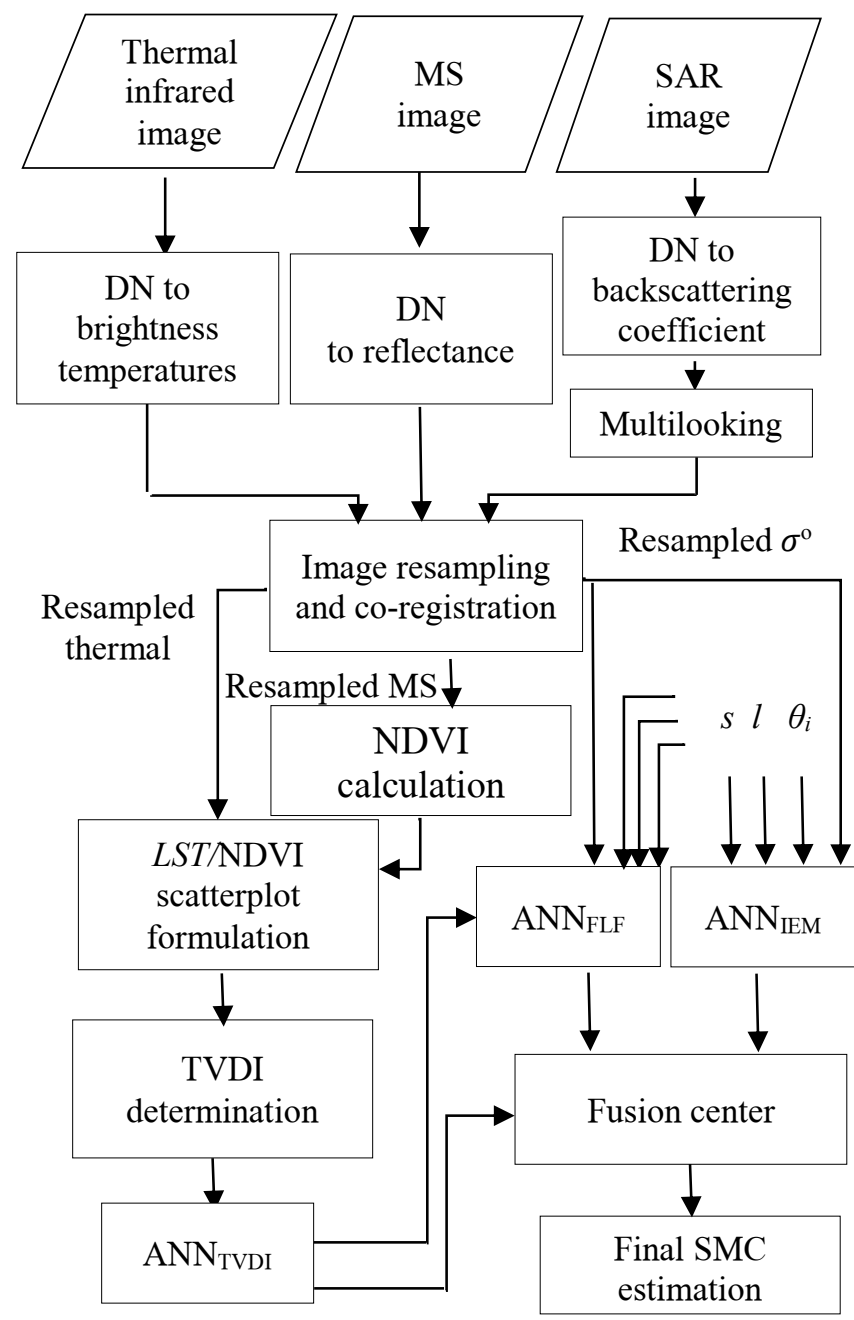

Figure 1: Flowchart of the proposed methodology.

The fusion center in the proposed methodology consists of a novel weights based estimation. Weights $\mathrm{w}_{1}, \mathrm{w}_{2}$ and $\mathrm{w}_{3}$ are assigned to output vectors (estimations) of $\mathrm{ANN}_{\mathrm{TVDI}}$, $A N N_{\text {IEM, }}$, and $A N N_{F L F}$ respectively. The final fused estimation is achieved by weighing each estimation as depicted in equation 1:

$$
S M C_{\text {fused }}=w_{1} S M C_{T V D I}+w_{2} S M C_{I E M}+w_{3} S M C_{F L F}
$$

where $\mathrm{SMC}_{\text {fused }}$ is the final fused estimation, $\mathrm{SMC}_{\mathrm{TVDI}}$ is the estimation derived from $\mathrm{ANN}_{\mathrm{TVDI}}, \mathrm{SMC}_{\mathrm{IEM}}$ is the estimation derived from $\mathrm{ANN}_{\mathrm{IEM}}, \mathrm{SMC}_{\mathrm{FLF}}$ is the estimation derived from $\mathrm{ANN}_{\mathrm{FLF}}$ and $\mathrm{w} 1+\mathrm{w} 2+\mathrm{w} 3=1$. The metric of accuracy in this study is the Root Mean Square Error (RMSE) which is represented in equation 2:

$$
R M S E=\sqrt{\sum_{i=1}^{N} \frac{1}{N}\left(P_{i}-O_{i}\right)^{2}}
$$

where $\mathrm{N}$ is the number of data samples, $\mathrm{P}_{\mathrm{i}}$ are the estimated SMC values and $\mathrm{O}_{\mathrm{i}}$ are the measured SMC values. The most straight forward weights assignment is when all weights are equal $\left(\mathrm{w}_{1}=\mathrm{w}_{2}=\mathrm{w}_{3}=1 / 3\right)$ which represents the mean. However, this assignment is not remotely accurate as the concerned estimations have varying degrees of accuracy under different conditions as described in section 2. The proposed method consists of determining RMSE for each possible combination of the weights and selecting the combination with the minimum RMSE. The method is implemented through a Matlab function that accepts $\mathrm{SMC}_{\mathrm{TVDI}}, \mathrm{SMC}_{\mathrm{IEM}}, \mathrm{SMC}_{\mathrm{FLF}}$ vectors as input and returns the fused estimation (in $\mathrm{SMC}_{\text {fused }}$ ), the weights values and the RMSE as output. The weights values are then saved for future applications into a database of weights for later use in case of lack of ground truth measurements.

\section{STUDY AREA}

The study area is an agricultural field in Blackwell farms ( $1.4 \mathrm{~km}^{2}$ in size) located in Guildford, the county town of Surrey in South East England with coordinates (lat:51.2372, lon:-0.6260). The size of this field is (295 $\mathrm{m} \mathrm{x} 308 \mathrm{~m})$ with minimal vegetation cover (NDVI $\geq 0.21$ ). Earth observation datasets of this study are generated from the Sentinel-1 and Landsat- 8 satellites. In this study, a Ground Range Detected (GRD) product acquired with the Interferometric Wide Swath (IWS) and VV polarization is used. First steps are the radiometric calibration and Multi-looking of Sentinel-1 images. Afterwards, these images are resampled to $30 \mathrm{~m}$ to match the Landsat-8 multispectral $(30 \mathrm{~m})$ and thermal images $(100 \mathrm{~m}$ resampled to $30 \mathrm{~m})$. Digital Numbers (DN) of the multispectral and thermal images are then transformed to reflectance and brightness temperature values respectively. The collected in-situ measurements for this study are SMC levels and surface heights with the latter being crucial to formulate soil surface roughness profile. SMC levels are measured using an ML3 Theta Probe Soil Moisture Sensor at the depth of $5 \mathrm{~cm}$ of soil surface. Then, to generate an SMC map, each pixel of the earth observation images requires $4 \mathrm{SMC}$ measurements at the corners of that pixel. Finally, for a better representation of the spatial variability of SMC levels, the mean of those measurements is used instead of a single point based measurement. In the end, 110 SMC measurements are collected with a mean of $31.320 \%$ and standard deviation of $2.146 \%$. Two soil surface roughness measurements $(s, l)$ are collected using a mechanical profilometer. 


\section{EXPERIMENTAL RESULTS}

Table 1 showcases the different methods of estimation as well as their corresponding accuracy:

\begin{tabular}{|l|c|}
\hline \multicolumn{1}{|c|}{ Estimation Method } & RMSE \\
\hline $\mathrm{SMC}_{\mathrm{TVDI}}$ & 1.892 \\
\hline $\mathrm{SMC}_{\mathrm{IEM}}$ & 1.565 \\
\hline $\mathrm{SMC}_{\mathrm{FLF}}$ & 1.091 \\
\hline $\mathrm{SMC}$ estimation when $\mathrm{w}_{1}=\mathrm{w}_{2}=0.5$ & 1.615 \\
\hline $\mathrm{SMC}_{\mathrm{C}}$ estimation when $\mathrm{w}_{1}=\mathrm{w}_{2}=\mathrm{w}_{3}=1 / 3$ & 1.343 \\
\hline $\mathrm{SMC}_{\text {fused }}$ (using only $\mathrm{w}_{1}$ and $\left.\mathrm{w}_{2}\right)$ & 1.553 \\
\hline $\mathrm{SMC}_{\text {fused }}$ & 1.090 \\
\hline
\end{tabular}

Table 1: comparison of the results of the estimation methods used in this study.

$\mathrm{SMC}_{\mathrm{TVDI}}$ estimation displays the highest RMSE (1.892\%) which could be explained by the low spatial resolution of Landsat- 8 thermal infrared band $(100 \mathrm{~m})$, not to mention that this particular dataset has not undergone any atmospheric correction. The multispectral sensor of Landsat8 has its own problems when it comes to its spatial resolution as well: while it is better than the thermal sensor $(30 \mathrm{~m})$, the mix of spectral information in a resolution cell of this size can affect the final estimation due the spatial variability of SMC. $\mathrm{SMC}_{\mathrm{IEM}}$ estimation fares better than its $\mathrm{SMC}_{\text {TVDI }}$ counterpart (1.565\%), this is justified by the low intensity of vegetation cover in the agricultural field. $\mathrm{SMC}_{\mathrm{FLF}}$ estimation shows an improvement in terms of accuracy than both the $\mathrm{SMC}_{\mathrm{TVDI}}$ and $\mathrm{SMC}_{\mathrm{IEM}}$ estimations $(1.091 \%)$, as discussed in [5]. SMC estimations when using the mean of $\mathrm{SMC}_{\mathrm{TVDI}}$ and $\mathrm{SMC}_{\mathrm{IEM}}(1.615 \%)$ and the mean of $\mathrm{SMC}_{\mathrm{TVDI}}, \mathrm{SMC}_{\text {IEM }}$ and $\mathrm{SMC}_{\mathrm{FLF}}(1.343 \%)$ while the latter performs better than the individual estimations of $\mathrm{SMC}_{\mathrm{TVDI}}$ and $\mathrm{SMC}_{\mathrm{IEM}}$, both are worse than $\mathrm{SMC}_{\mathrm{FLF}}$. $\mathrm{SMC}_{\text {fused }}$ (using only $\mathrm{w}_{1}$ and $\mathrm{w}_{2}$ ) estimation $(1.553 \%)$ proves that the addition of $\mathrm{SMC}_{\mathrm{FLF}}$ estimation to the fusion method improves the accuracy of the final fusion as $\mathrm{SMC}_{\text {fused }}$ produces the best SMC estimation (1.090 \%). The fusion center consistently improves the accuracy of the final estimation in this dataset and the one in [5], the improvement varied and for this particular dataset it was minuscule.

\section{CONCLUSION}

This study proposed a novel weight based decision level fusion method of Landsat-8 and Sentinel-1 for SMC estimations. Tests and analysis have shown that this weight based estimation produces the best result out of all the tested methods (lowest RMSE $=1.090 \%$ ). The improvement is not sizeable $\left(0.001 \%\right.$ lower than $\left.\mathrm{SMC}_{\mathrm{FLF}}\right)$, further investigations to validate this improvement are necessary to build a weights database capable of reliably estimating SMC values without reliance on ground truth data.

\section{REFERENCES}

[1] K. E. Trenberth et al, "Global warming and changes in drought," Nature Climate Change, vol. 4, (1), 2014.

[2] V. Naeimi et al, "An improved soil moisture retrieval algorithm for ERS and METOP scatterometer observations," IEEE Trans. Geosci. Remote Sens., vol. 47, (7), 2009.

[3] M. E. Holzman, R. Rivas and M. C. Piccolo, "Estimating soil moisture and the relationship with crop yield using surface temperature and vegetation index," International Journal of Applied Earth Observation and Geoinformation, vol. 28, pp. 181-192, 2014.

[4] J. Dong et al, "Advances in multi-sensor data fusion: Algorithms and applications," Sensors, vol. 9, (10), 2009.

[5] O.Yahia, R.Guida and P.Iervolino, "Sentinel-1 and landsat-7 ETM+ feature level fusion for soil moisture content estimation," in 2018, .

[6] M. T. Hallikainen et al, "Microwave dielectric behavior of wet soil-part 1: Empirical models and experimental observations," IEEE Trans. Geosci. Remote Sens., (1), 1985.

[7] S. Khabazan, M. Motagh and M. Hosseini, "Evaluation of Radar Backscattering Models IEM, OH, and Dubois using $\mathrm{L}$ and C-Bands SAR Data over different vegetation canopy covers and soil depths," ISPRS-International Archives of the Photogrammetry, Remote Sensing and Spatial Information Sciences, pp. 225-230, 2013.

[8] I. Sandholt, K. Rasmussen and J. Andersen, "A simple interpretation of the surface temperature/vegetation index space for assessment of surface moisture status," Remote Sens. Environ., vol. 79, (2), pp. 213-224, 2002.

[9] N. Baghdadi, S. Gaultier and C. King, "Retrieving surface roughness and soil moisture from synthetic aperture radar (SAR) data using neural networks," Canadian Journal of Remote Sensing, vol. 28, (5), pp. 701-711, 2002.

[10] A. Ross and R. Govindarajan, "Feature level fusion using hand and face biometrics," in Proceedings of SPIE Conference on Biometric Technology for Human Identification II, 2005,

[11] D. D. Alexakis et al, "Soil moisture content estimation based on Sentinel-1 and auxiliary earth observation products. A hydrological approach," Sensors, vol. 17, (6) 2017. 